\title{
Early warnings and missed alarms for abrupt monsoon transitions
}

\author{
Z. A. Thomas ${ }^{1,2}$, F. Kwasniok ${ }^{3}$, C. A. Boulton ${ }^{2}$, P. M. Cox $^{3}$, R. T. Jones ${ }^{2}$, T. M. Lenton ${ }^{2}$, and C. S. M. Turney ${ }^{1}$ \\ ${ }^{1}$ Climate Change Research Centre and School of Biological, Earth \& Environmental Sciences, University of \\ New South Wales, Sydney, NSW 2052, Australia \\ ${ }^{2}$ College of Life and Environmental Sciences, University of Exeter, Exeter, EX4 4RJ, UK \\ ${ }^{3}$ College of Engineering, Mathematics and Physical Sciences, University of Exeter, Exeter, EX4 4QF, UK
}

Correspondence to: Z. A. Thomas (z.thomas@unsw.edu.au)

Received: 23 March 2015 - Published in Clim. Past Discuss.: 13 April 2015

Revised: 15 November 2015 - Accepted: 16 November 2015 - Published: 8 December 2015

\begin{abstract}
Palaeo-records from China demonstrate that the East Asian Summer Monsoon (EASM) is dominated by abrupt and large magnitude monsoon shifts on millennial timescales, switching between periods of high and weak monsoon rains. It has been hypothesized that over these timescales, the EASM exhibits two stable states with bifurcation-type tipping points between them. Here we test this hypothesis by looking for early warning signals of past bifurcations in speleothem $\delta^{18} \mathrm{O}$ records from Sanbao Cave and Hulu Cave, China, spanning the penultimate glacial cycle. We find that although there are increases in both autocorrelation and variance preceding some of the monsoon transitions during this period, it is only immediately prior to the abrupt monsoon shift at the penultimate deglaciation (Termination II) that statistically significant increases are detected. To supplement our data analysis, we produce and analyse multiple model simulations that we derive from these data. We find hysteresis behaviour in our model simulations with transitions directly forced by solar insolation. However, signals of critical slowing down, which occur on the approach to a bifurcation, are only detectable in the model simulations when the change in system stability is sufficiently slow to be detected by the sampling resolution of the data set. This raises the possibility that the early warning "alarms" were missed in the speleothem data over the period 224-150 kyr and it was only at the monsoon termination that the change in the system stability was sufficiently slow to detect early warning signals.
\end{abstract}

\section{Introduction}

The Asian Summer Monsoon directly influences over $60 \%$ of the world's population (Wu et al., 2012) and yet the drivers of past and future variability remain highly uncertain (Zickfeld et al., 2005; Levermann et al., 2009). Evidence from radiometrically dated East Asian speleothem records of past monsoon behaviour (Yuan et al., 2004) suggests that on millennial timescales, the EASM is driven by a $23 \mathrm{kyr}$ precession cycle (Kutzbach, 1981; Wang et al., 2008), but also influenced by feedbacks in sea surface temperatures and changing boundary conditions including Northern Hemisphere ice volume (An, 2000; Sun et al., 2015). The abrupt nature of the monsoon behaviour (interpreted as a precipitation proxy from $\delta^{18} \mathrm{O}$ values from Chinese speleothem records; see Sect. 1.4) in comparison to the sinusoidal insolation forcing strongly implies that this response is nonlinear (Fig. 1); whilst Northern Hemisphere summer insolation (NHSI) follows a quasi-sinusoidal cycle, the $\delta^{18} \mathrm{O}$ profile in speleothems exhibits a step function, suggesting the presence of threshold behaviour in the monsoon system (Schewe et al., 2012). Though the vulnerability of society has clearly changed, future abrupt monsoon shifts, whether caused by orbital or anthropogenic forcing, are likely to have major devastating societal impacts (Donges et al., 2015).

A minimum conceptual model of the East Asian Summer Monsoon developed by Zickfeld et al. (2005), stripped down by Levermann et al. (2009) and updated by Schewe et al. (2012), shows a non-linear solution structure with thresholds for switching a monsoon system between "on" or "off" states that can be defined in terms of atmospheric humidity - 


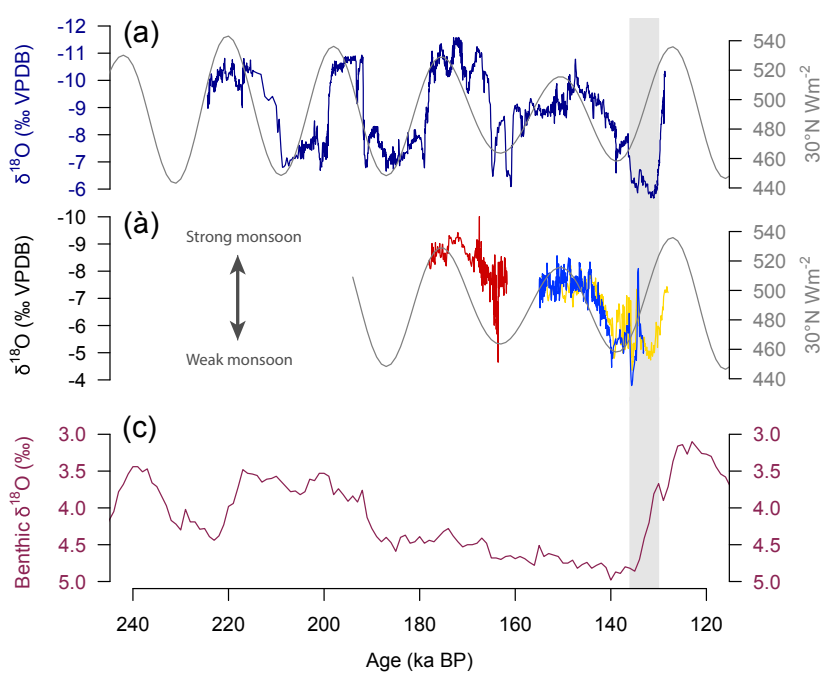

Figure 1. (a) Northern Hemisphere summer insolation (NHSI) in June at $30^{\circ} \mathrm{N}$ (Berger and Loutre, 1991; grey), $\delta^{18} \mathrm{O}$ speleothem data from Sanbao Cave (Wang et al., 2008; dark blue), (b) $\delta^{18} \mathrm{O}$ speleothem data from Hulu Cave (Wang et al., 2001); speleothem MSH (red), MSP (blue) and MSX (yellow), (c) $\delta^{18} \mathrm{O}$ per mille benthic carbonate (Lisiecki and Raymo, 2005; proxy for global ice volume; purple). The grey shaded area indicates the Weak Monsoon Interval prior to Termination II.

in particular, atmospheric specific humidity over the adjacent ocean (Schewe et al., 2012). Critically, if specific humidity levels pass below a certain threshold, for instance, as a result of reduced sea surface temperatures, insufficient latent heat is produced in the atmospheric column and the monsoon fails. This moisture-advection feedback allows for the existence of two stable states, separated by a saddle-node bifurcation (Zickfeld et al., 2005; although interestingly, the conceptual models of Levermann et al. (2009) and Schewe et al. (2012) are characterized by a single bifurcation point for switching "off" the monsoon and an arbitrary threshold to switch it back "on"). Crucially, the presence of a critical threshold at the transition between the strong and weak regimes of the EASM means that early warning signals related to "critical slowing down" (Dakos et al., 2008; Lenton et al., 2012) could be detectable in suitable proxy records.

The aim of this study is twofold: (1) to test whether shifts in the EASM during the penultimate glacial cycle (Marine Isotope Stage 6) are consistent with bifurcational tipping points, and (2) if so, is it possible to detect associated early warning signals? To achieve this, we analyse two $\delta^{18} \mathrm{O}$ speleothem records from China, and construct a simple model that we derive directly from these data to test whether we can detect early warning signals of these transitions.

\subsection{Detecting early warning signals}

We perform "tipping point analysis" on both the $\delta^{18} \mathrm{O}$ speleothem records and on multiple simulations derived from our model. This analysis aims to find early warning signs of impending tipping points that are characterized by a bifurcation (rather than a noise-induced tipping, induced by stochastic fluctuations with no change in forcing control, or rate-dependent tipping, where a system fails to track a continuously changing quasi-static attractor e.g. Ashwin et al., 2012). These tipping points can be mathematically detected by looking at the pattern of fluctuations in the short-term trends of a time series before the transition takes place. A phenomenon called "critical slowing down" occurs on the approach to a tipping point, whereby the system takes longer to recover from small perturbations (Kleinen et al., 2003; Held and Kleinen, 2004; Dakos et al., 2008). This longer recovery rate causes the intrinsic rates of change in the system to decrease, which is detected as a short-term increase in the autocorrelation or "memory" of the time series (Ives, 1995), often accompanied by an increasing trend in variance (Lenton et al., 2012). It has been theoretically established that autocorrelation and variance should both increase together (Ditlevsen and Johnsen, 2010; Thompson and Sieber, 2011). Importantly, it is the increasing trend, rather than the absolute values of the autocorrelation and variance that indicate critical slowing down. Detecting the phenomenon of critical slowing down relies on a timescale separation, whereby the timescale forcing the system is much slower than the timescale of the system's internal dynamics, which is in turn much longer than the frequency of data sampling the system (Held and Kleinen, 2004). Importantly, the monsoon transitions span hundreds of years (corresponding to several data points), meeting the criterion that the frequency of sampling is higher than the timescale of the transition of the system.

\subsection{Missed alarms}

Although efforts have been taken to reduce the chances of type I (incorrect rejection of a true null hypothesis, otherwise known as a "false positive") and type II (failure to reject a false null hypothesis, or "false negative") errors by correct pre-processing of data e.g. (Lenton, 2011), totally eradicating the chances of false positive and false negative results remains a challenge (Scheffer, 2010; Lenton et al., 2012; Dakos et al., 2014). Type II errors or "missed alarms", as discussed in Lenton (2011), may occur when internal noise levels are such that the system is "tipped" into a different state prior to reaching the bifurcation point, precluding the detection of early warning signals. Type I errors are potentially easier to guard against by employing strict protocols by which to reject a null hypothesis.

\subsection{Using speleothem $\delta^{18} \mathrm{O}$ data as a proxy of past monsoon strength}

Highly-resolved $\left(\sim 10^{2}\right.$ years $)$ and precisely dated speleothem records of past monsoonal variability are well placed to test for early warning signals. The use of 
speleothem-based proxies to reconstruct patterns of palaeomonsoon changes has increased rapidly over recent decades with the development of efficient sampling and dating techniques. However, there is currently some debate surrounding the climatic interpretation of Chinese speleothem $\delta^{18} \mathrm{O}$ records (An et al., 2015), which can be influenced by competing factors that affect isotope fractionation. The oxygen isotopic composition of speleothem calcite is widely used to reconstruct palaeohydrological variations due to the premise that speleothem calcite $\delta^{18} \mathrm{O}$ records the stable isotopic content of precipitation, which has been shown to be inversely correlated with precipitation amount (Dansgaard, 1964; Lee and Swann, 2010), a relationship known as the "amount effect". Although the $\delta^{18} \mathrm{O}$ of speleothem calcite in China has traditionally been used as a proxy for the "amount effect" (Cheng et al., 2006, 2009; Wang et al., 2008, 2009), this has been challenged by other palaeo-wetness proxies, notably Maher (2008), who argues that speleothems may be influenced by changes in rainfall source rather than amount. The influence of the Indian Monsoon has also been proposed as an alternative cause for abrupt monsoon variations in China (Liu et al., 2006; Pausata et al., 2011), though this has since been disputed (Wang and Chen, 2012; Liu et al., 2014). Importantly, however, robust replications of the same $\delta^{18} \mathrm{O}$ trends in speleothem records across the wider region suggest they principally represent changes in the delivery of precipitation $\delta^{18} \mathrm{O}$ associated with the EASM (Cheng et al., 2009, 2012; Li et al., 2014; Duan et al., 2014; Liu et al., 2014; Baker et al., 2015).

Specific data requirements are necessary to search for early warning signs of tipping points in climate systems; not only does the data have to represent a measure of climate, it also must be of a sufficient length and resolution to enable the detection of critical slowing down. In addition, since time series analysis methods require interpolation to equidistant data points, a relative constant density of data points is important, so that the interpolation does not skew the data. The speleothem $\delta^{18} \mathrm{O}$ records that we have selected to fulfil these criteria, as described in more detail in Sect. 2.1.

\section{Methods}

\subsection{Data selection}

We used the Chinese speleothem sequences from Sanbao Cave $\left(31^{\circ} 40^{\prime} \mathrm{N}, 110^{\circ} 26^{\prime} \mathrm{E}\right.$; Wang et al., 2008) and Hulu Cave $\left(32^{\circ} 30^{\prime} \mathrm{N}, 119^{\circ} 10^{\prime} \mathrm{E}\right.$; Wang et al., 2001) to search for early warning signals. Sanbao Cave (speleothem SB11) and Hulu Cave (speleothem MSP) have two of the highest resolution chronologies in the time period of interest, with a relatively constant density of data points, providing some of the best records of Quaternary-scale monsoonal variation. Speleothem $\delta^{18} \mathrm{O}$ records offer considerable advantages for investigating past changes in the EASM: their long duration $\left(10^{3}-10^{4}\right.$ years $)$, high-resolution $(\sim 100$ years $)$ and pre-

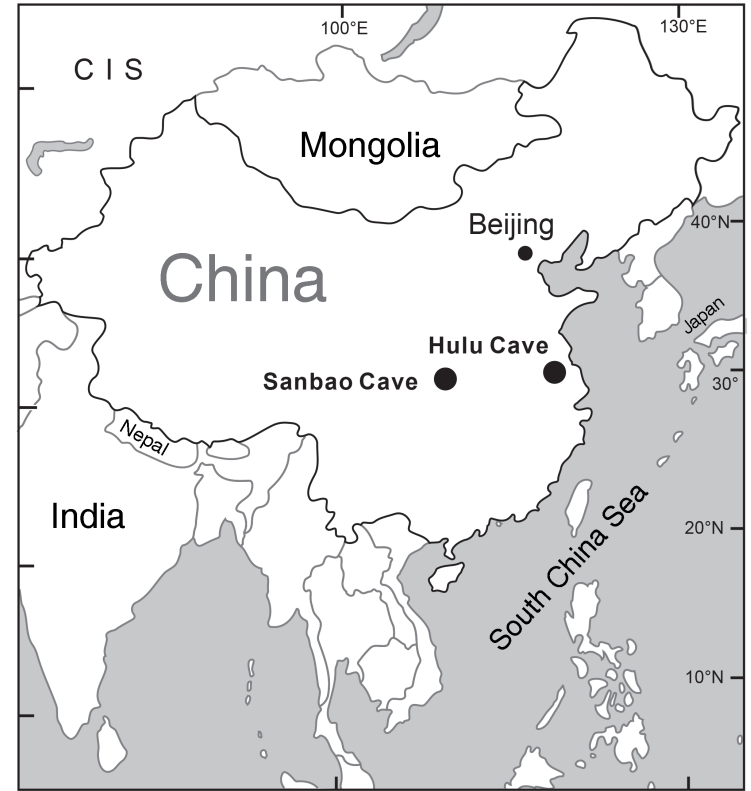

Figure 2. Map showing the location of Sanbao and Hulu caves.

cise and absolute-dated chronologies (typically $1 \mathrm{kyr}$ at $1 \sigma$ ), make them ideal for time series analysis. Speleothem SB11 has one of the longest, continuous $\delta^{18} \mathrm{O}$ records in China, and is the only series spanning an entire glacial cycle without using a spliced record (Wang et al., 2008). Speleothem MSP has a comparable resolution and density to SB11, though is significantly shorter. Crucially, the cave systems lie within two regionally distinct areas (Fig. 2), indicating that parallel changes in $\delta^{18} \mathrm{O}$ cannot be explained by local effects.

\subsection{Searching for bimodality}

A visual inspection of a histogram of the speleothem $\delta^{18} \mathrm{O}$ data was initially undertaken to determine whether the data are likely to be bimodal. We then applied a Dip-test of unimodality (Hartigan and Hartigan, 1985) to test whether our data are bimodal. To investigate further the dynamical origin of the modality of our data we applied non-stationary potential analysis (Kwasniok, 2013, 2015). A non-stationary potential model (discussed in more detail in Sect. 2.4) was fitted, modulated by the solar forcing (NHSI June $30^{\circ} \mathrm{N}$ ), covering the possibility of directly forced transitions as well as noise-induced transitions with or without stochastic resonance.

\subsection{Tipping point analysis}

A search for early warning signals of a bifurcation at each monsoon transition was carried out between 224-128 kyr of the Sanbao Cave and Hulu Cave speleothem records. Stable periods of the Sanbao Cave $\delta^{18} \mathrm{O}$ record (e.g. excluding the abrupt transitions) were initially identified visually 
and confirmed by subsequent analysis using a climate regime shift detection method described by Rodionov (2004). Data pre-processing involved removal of long-term trends using a Gaussian kernel smoothing filter and interpolation to ensure that the data are equidistant (a necessary assumption for time series analysis), before the trends in autocorrelation and variance (using the $R$ functions acf and var respectively) are measured over a sliding window of half the data length (Lenton et al., 2012). The density of data points over time do not change significantly in either record and thus the observed trends in autocorrelation are not an artefact of the data interpolation. The smoothing bandwidth was chosen such that long-term trends were removed without overfitting the data. A sensitivity analysis was undertaken by varying the size of the smoothing bandwidth and sliding window to ensure the results were robust over a range of parameter choices. The nonparametric Kendall's tau rank correlation coefficient was applied (Kendall, 1948; Dakos et al., 2008) to test for statistical dependence for a sequence of measurements against time, varying between +1 and -1 , describing the sign and strength of any trends in autocorrelation and variance.

\subsubsection{Assessing significance}

The results were tested against surrogate time series to ascertain the significance level of the results found, based on the null hypothesis that the data are generated by a stationary Gaussian linear stochastic process. This method for assessing significance of the results is based on Dakos et al. (2012a). The surrogate time series were generated by randomizing the original data over 1000 permutations, which is sufficient to adequately estimate the probability distribution of the null model, and destroys the memory while retaining the amplitude distribution of the original time series. The autocorrelation and variance for the original and each of the surrogate time series was computed, and the statistical significance obtained for the original data by comparing against the frequency distribution of the trend statistic (Kendall tau values of autocorrelation and variance) from the surrogate data. Importantly, the Kendall tau values are calculated relatively, thus when the autocorrelation is destroyed by randomization, the null model distribution does not change. Higher Kendall tau values indicate a stronger increasing trend. The 90th and 95th percentiles provided the 90 and $95 \%$ rejection thresholds (or $p$ values of 0.1 and 0.05 ) respectively. According to the fluctuation-dissipation theorem (Ditlevsen and Johnsen, 2010), both autocorrelation and variance should increase together on the approach to a bifurcation. Previous tipping point literature has often used a visual increasing trend of autocorrelation and variance as indicators of critical slowing down. Although using surrogate data allows a quantitative assessment of the significance of the results, there is no consensus on what significance level is necessary to declare the presence of precursors of critical slowing down. To guard against type I errors, we determine for this study that "statistically significant" early warning indicators occur with increases in both autocorrelation and variance with $p$ values $<0.1$. We have chosen this benchmark in line with previous studies using a similar null model that have described results with $p<0.1$ as "robust" (Dakos et al., 2008; Boulton and Lenton, 2015).

\subsection{Non-stationary potential analysis}

To supplement the analysis of the speleothem records and help interpret the results, a simple stochastic model derived directly from the Sanabo cave $\delta^{18} \mathrm{O}$ data was constructed. Non-stationary potential analysis (Kwasniok, 2013, 2015) is a method for deriving from time series data a simple dynamical model which is modulated by external factors, here solar insolation. The technique allows extraction of basic dynamical mechanisms and to distinguish between competing dynamical explanations.

The dynamics of the monsoon system are conceptually described as noise-driven motion in a time-dependent potential landscape. The governing equation is a one-dimensional nonstationary effective Langevin equation:

$\dot{x}=-V^{\prime}(x ; t)+\sigma \eta$.

The model variable $x$ is identified with the speleothem $\delta^{18} \mathrm{O}$ record, which is a proxy for monsoon strength. The potential function $V(x ; t)$ describes the force field governing the monsoon system. $\eta$ is a white Gaussian noise process with zero mean and unit variance, and $\sigma$ is the amplitude of the stochastic forcing. The noise term is meant to account for the influence of unresolved temporal and spatial scales. The potential landscape is time-dependent, modulated by the solar insolation:

$V(x ; t)=U(x)+\gamma I(t) x$.

The time-independent part of the potential is modelled by a fourth-order polynomial, allowing for possible bi-stability (Kwasniok and Lohmann, 2009):

$U(x)=\sum_{i=1}^{4} a_{i} x^{i}$.

$I(t)$ is the insolation forcing and $\gamma$ is a coupling parameter. The modulation of the potential is only in the linear term, that is, the time-independent potential system is subject to the scaled insolation forcing $\gamma I(t)$. The insolation is represented as a superposition of three main frequencies as

$I(t)=\alpha_{0}+\sum_{i=1}^{3}\left[\alpha_{i} \cos \left(\frac{2 \pi t}{T_{i}}\right)+\beta_{i} \sin \left(\frac{2 \pi t}{T_{i}}\right)\right]$

with time $t$ measured in kyr. The expansion coefficients $\alpha_{i}$ and $\beta_{i}$ are determined by least-squares regression on the insolation time series over the time interval of the speleothem 

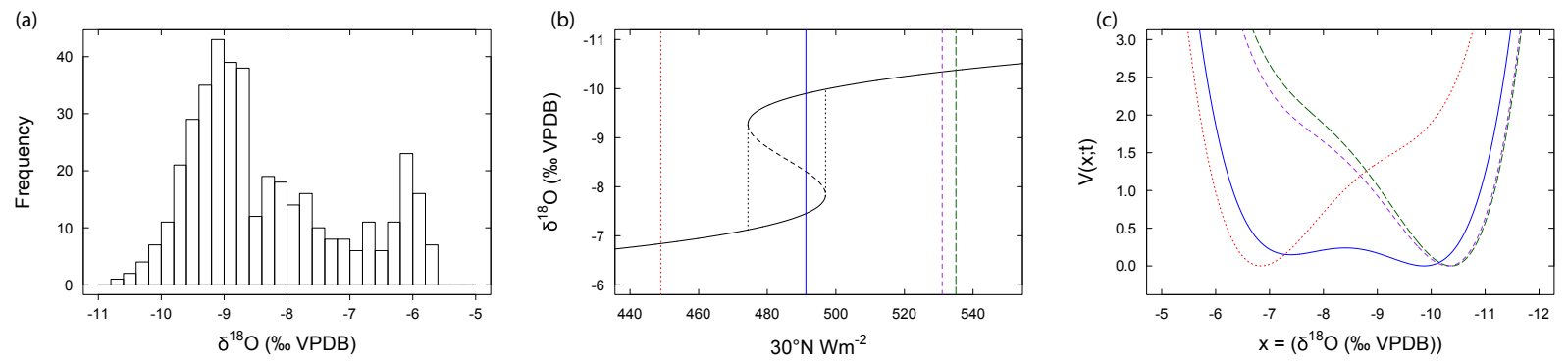

Figure 3. (a) Histogram showing the probability density of the speleothem data aggregated over 224-128 kyr, (b) bifurcation diagram obtained from potential model analysis, showing bi-stability and hysteresis. Solid black lines indicate stable states, dashed line unstable states, and dotted vertical lines the jumps between the two stable branches. Coloured vertical lines correspond to the insolation values for which the potential curve is shown in panel (c); (c) shows how the shape of the potential well changes over one transition cycle (198-175 kyr) (green long dash $=535 \mathrm{~W} \mathrm{~m}^{-2}$, purple short dash $=531 \mathrm{~W} \mathrm{~m}^{-2}$, blue solid $=491 \mathrm{~W} \mathrm{~m}^{-2}$, red dotted $=449 \mathrm{~W} \mathrm{~m}{ }^{-2}$; for more details see Fig. 10).

record. The periods $T_{i}$ are found by a search over a grid with mesh size $0.5 \mathrm{kyr}$. They are, in order of decreasing contribution $\alpha_{i}^{2}+\beta_{i}^{2}, T_{1}=23 \mathrm{kyr}, T_{2}=19.5 \mathrm{kyr}$ and $T_{3}=42 \mathrm{kyr}$. This yields an excellent approximation of the insolation time series over the time interval under consideration here.

The potential model covers and allows us to distinguish between two possible scenarios: (i) in the bifurcation scenario, the monsoon transitions are directly forced by the insolation, where two states are stable in turn, one at a time. This corresponds to a fairly large value of $\gamma$. (ii) Alternatively, two stable states could be available at all times with noise-induced switching between them. This is realized with $\gamma=0$, giving a stationary potential. The height of the potential barrier separating the two states could be modulated by the insolation, possibly giving rise to a stochastic resonance which would explain the high degree of coherence between the solar forcing and the monsoon transitions. The latter variant would correspond to a small but non-zero value of $\gamma$.

The shape of the potential, as well as the noise level, are estimated directly from the speleothem data according to the maximum likelihood principle. We take a two-step approach, combining non-stationary probability density modelling (Kwasniok, 2013) and dynamical modelling (Kwasniok, 2015). The shape of the potential is estimated from the probability density of the data. The quasi-stationary probability density of the potential model is

$p(x ; t)=Z^{-1}(t) \exp \left[-\frac{2 V(x ; t)}{\sigma^{2}}\right]$,

with a time-dependent normalization constant $Z(t)$. The coefficients $a_{i}$ and the coupling constant $\gamma$ are estimated by maximizing the likelihood function

$L\left(x_{1}, \ldots, x_{N}\right)=\prod_{i=1}^{N} p\left(x_{n} ; t_{n}\right)$,

as described in Kwasniok (2013). The size of the data set is $N=1288$. This leaves the noise level undetermined as a scaling of the potential with a constant $c$ and a simultaneous scaling of the noise variance with $c$ keeps the quasistationary probability density unchanged. We set $\sigma=1$ for the (preliminary) estimation of $a_{i}$ and $\gamma$. The noise level is now determined from the dynamical likelihood function based on the time evolution of the system (Kwasniok, 2015). The Langevin equation is discretised according to the EulerMaruyama scheme:

$x_{n+1}=x_{n}-\delta t_{n} V^{\prime}\left(x_{n} ; t_{n}\right)+\sqrt{\delta t_{n}} \sigma \eta_{n}$.

The sampling interval of the data is $\delta t_{n}=t_{n+1}-t_{n}$. The loglikelihood function of the data is

$$
\begin{aligned}
& l\left(x_{1}, \ldots, x_{N} \mid x_{0}\right)=-\frac{N}{2} \log 2 \pi-N \log \sigma-\frac{1}{2} \\
& \sum_{n=0}^{N-1}\left(\log \delta t_{n}+\frac{\left[x_{n+1}-x_{n}+\delta t_{n} V^{\prime}\left(x_{n} ; t_{n}\right)\right]^{2}}{\delta t_{n} \sigma^{2}}\right) .
\end{aligned}
$$

The scaling constant $c$ is searched on a grid with mesh size 0.01 and the log-likelihood maximized, giving the final estimates of all parameters. Both estimation procedures are applied directly to the unevenly sampled data without any prior interpolation. We remark that the more natural and simpler approach of estimating all parameters simultaneously from the dynamical likelihood (Kwasniok, 2015) here yields a negative leading-order coefficient $a_{4}$ and thus the model cannot be integrated over a longer time period without the trajectory escaping to infinity. This possibly points at limitations in the degree of validity of the one-dimensional potential model. Palaeoclimatic records reflect a multitude of complex processes and any model as simple as Eq. (1) cannot be expected to be more than a skeleton model used to pinpoint and contrast basic dynamical mechanisms. The described estimation method guarantees a positive leading-order coefficient $a_{4}$ and therefore a globally stable model.

It has been suggested that the EASM system responds specifically to 21 July insolation at $65^{\circ} \mathrm{N}$ with a "near-zero 


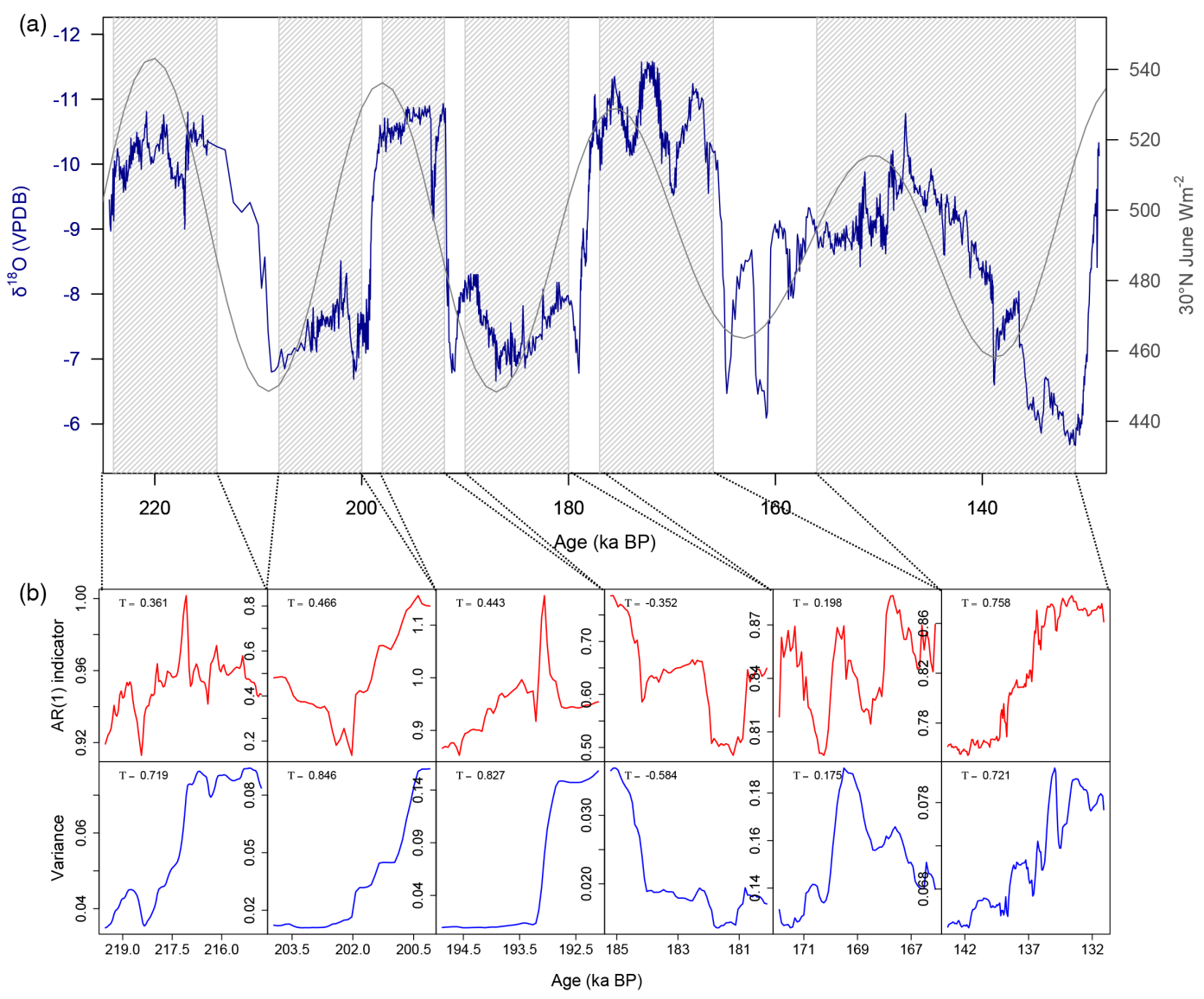

Figure 4. (a) $\delta^{18} \mathrm{O}$ speleothem data from Sanbao Cave (SB11; blue line) and NHSI in June at $30^{\circ} \mathrm{N}$ (grey line). Grey hatched areas show the sections of data selected for tipping point analysis. (b) Autocorrelation and variance for each period prior to a transition.

phase lag" (Ruddiman, 2006). However, given that EASM development is affected by both remote and local insolation forcing (Liu et al., 2006), we use an insolation latitude local to the Sanbao Cave record, consistent with earlier studies from this and other speleothem sequences (Wang et al., 2001). Since the monthly maximum insolation shifts in time with respect to the precession parameter, the $30^{\circ} \mathrm{N}$ June insolation was used, though we acknowledge that the insolation changes of $65^{\circ} \mathrm{N} 21$ July as used by Wang et al. (2008) are similar with regard to the timing of maxima and minima. Crucially, immediately prior to Termination II, the Chinese speleothem data (including Sanbao Cave) record a "Weak Monsoon Interval" between 135.5 and $129 \mathrm{kyr}$ (Cheng et al., 2009), suggesting a lag of approximately $6.5 \mathrm{kyrs}$ following Northern Hemisphere summer insolation (Fig. 1).

Having derived a model from the data, 100 realizations were analysed to test whether early warning signals could be detected in the model output, using the methods set out in Sect. 2.3. We initially chose the sampling resolution of the model outputs to be comparable to the speleothem data $\left(10^{2}\right.$ years). Subsequently, the model was manipulated by changing both the noise level and the sampling resolution in order to explore the effect of these on the early warning signals in a hypothetical scenario. To enable a straightforward comparison of the rate of forcing and the sampling resolution we linearized the solar insolation using the minimum and maximum values of the solar insolation over the time span of the model (224-128 kyr). This approach was preferred rather than using a sinusoidal forcing since early warning signals are known to work most effectively when there is a constant increase in the forcing. To detrend the time series data, we ran the model without any external noise forcing to obtain the equilibrium solution to the system, which we then subtracted from the time series, which did include noise. In addition, we manipulated the noise level of the model by altering the amplitude of the stochastic forcing ( $\sigma$ in Eq. 1). The time step in the series was reduced so that 6000 time points were available prior to the bifurcation and to ensure no data from beyond the tipping point was included in the analysis. Sampling the same time series at different resolutions allowed us to explore the effect of this on the early warning signals. When comparing early warning signals for differing sample steps and noise levels, the same iteration of the model was used to enable a direct comparison. 

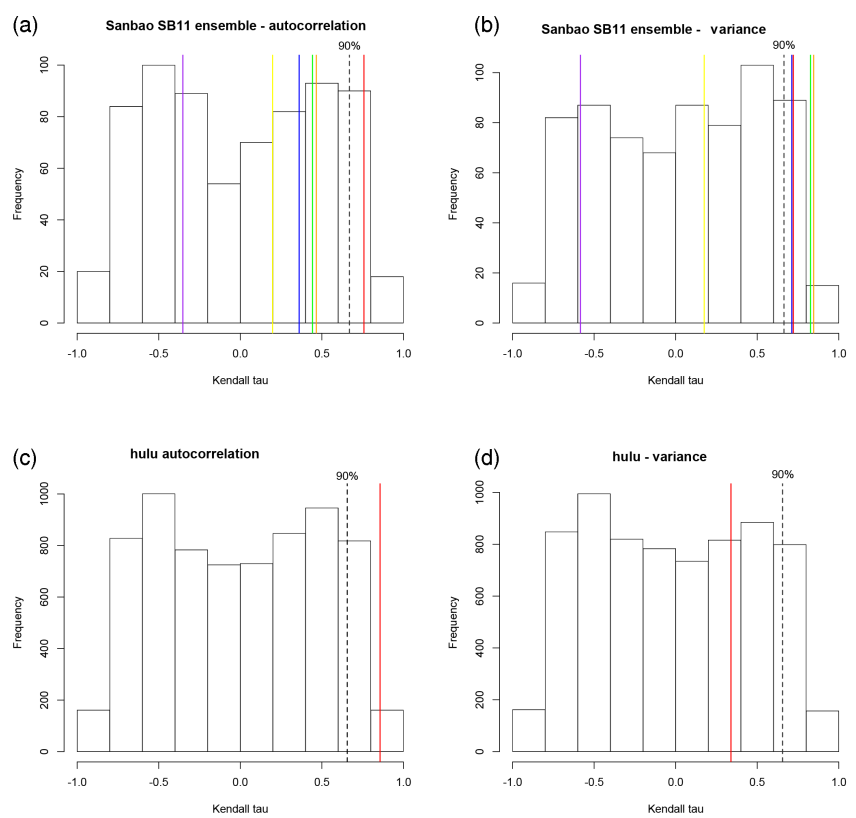

Figure 5. Histogram showing frequency distribution of Kendall tau values from 1000 realizations of a surrogate time series model (described in Sect. 2.3.1), for Sanbao Cave (a, b) and Hulu Cave (c, d) $\delta^{18} \mathrm{O}$ data. The grey dashed lines indicate the $90 \%(p<0.1)$ and $95 \%(p<0.05)$ significance level. Each coloured line denotes the Kendall tau values for autocorrelation and variance, for each section of speleothem data analysed (red $=131-156 \mathrm{kyr}$; yellow $=166-177 \mathrm{kyr}$; purple $=180-189 \mathrm{kyr}$; green $=191-198 \mathrm{kyr}$; orange $=200-208 \mathrm{kyr}$; blue $=214-225 \mathrm{kyr}$ ).

\section{Results}

\subsection{Bimodality and non-stationary potential modelling}

A histogram of $\delta^{18} \mathrm{O}$ values suggests there are two modes in the EASM between 224-128 kyr, as displayed by the double peak structure in Fig. 3a, supporting a number of studies that observe bimodality in tropical monsoon systems (Zickfeld et al., 2005; Schewe et al., 2012). We also apply a Dip-test of unimodality (Hartigan and Hartigan, 1985) and find that our null hypothesis of unimodality is rejected $(D=0.018$, $p=0.0063$ ) and thus our data are at least bimodal. To investigate further the dynamical origin of this bimodality we applied non-stationary potential analysis (Kwasniok, 2013, 2015). This showed a bi-stable structure to the EASM with hysteresis (Fig. 3b, c), suggesting that abrupt monsoon transitions may involve underlying bifurcations. The monsoon transitions appear to be predominantly directly forced by the insolation. There is a phase in the middle of the transition cycle between the extrema of the insolation where two stable states are available at the same time but this phase is too short for noise-induced switches to play a significant role.

We are able to clearly refute from the speleothem data the scenario of noise-induced switching between two simultaneously available states in favour of the bifurcation sce- nario. When fitting a model without solar insolation forcing (that is, $\gamma=0$ ) we obtain a stationary potential with two deep wells and noise-driven switching between them. However, the pdf-based log-likelihood of Eq. (6) is $l=-2149.1$ vs. $l=-1943.2$ for the model with insolation forcing and the dynamical log-likelihood of Eq. (8) is $l=-353.6$ vs. $l=-346.6$. This provides very strong evidence for the bifurcation scenario; based on both likelihood functions, both the Akaike and the Bayesian information criterion clearly prefer the model with solar insolation forcing. The value of $\gamma$ is fairly large and the stationary part of the potential is not strongly bistable, as evidenced by the shape of the potential given in Fig. 3, ruling out the stochastic resonance scenario. The uncertainty in all parameters, including the noise level, is very small, making our model estimation robust. We tried more complicated models where also the higher-order terms in the potential are modulated by the insolation rather than just the linear term or where the solar insolation enters nonlinearly into the model; the gain in likelihood is found to be rather minor compared to the gain achieved when adding the modulation in the linear term of the potential.

\subsection{Tipping point analysis}

We applied tipping point analysis on the Sanbao Cave $\delta^{18} \mathrm{O}$ record on each section of data prior to a monsoon transition. Although autocorrelation and variance do increase prior to some of the abrupt monsoon transitions (Fig. 4), these increases are not consistent through the entire record. Surrogate data sets used to test for significance of our results showed that $p$ values associated with these increases are only $<0.1$ for both autocorrelation and variance (Fig. 5) in one instance. Although a visual increasing trend has been used in previous literature as an indicator of critical slowing down, we choose more selective criteria to guard against the possibility of false positives.

The only section of data prior to a monsoon transition that sees $p$ values of $<0.1$ for the increases in both autocorrelation and variance is for the data spanning the period 150 to $129 \mathrm{kyr}$ in the Sanbao Cave record, before Monsoon Termination II (Fig. 6). We find that the Kendall tau value for autocorrelation has a significance level of $p<0.05$ and for variance a significance level of $p<0.1$ (Fig. 5a and b). These proportional positive trends in both autocorrelation and variance are consistent with critical slowing down on the approach to a bifurcation (Ditlevsen and Johnsen, 2010).

To test whether the signal is present in other EASM records, we undertook the same analysis on a second speleothem sequence of comparable age (Fig. 7). We find that speleothem MSP from Hulu Cave $\left(32^{\circ} 30^{\prime} \mathrm{N}, 119^{\circ} 10^{\prime} \mathrm{E}\right.$; Wang et al., 2001) displays a comparable increase in autocorrelation and variance to speleothem SB11 from Sanbao Cave, though these do display slightly lower $p$ values (Fig. 5c and d). 

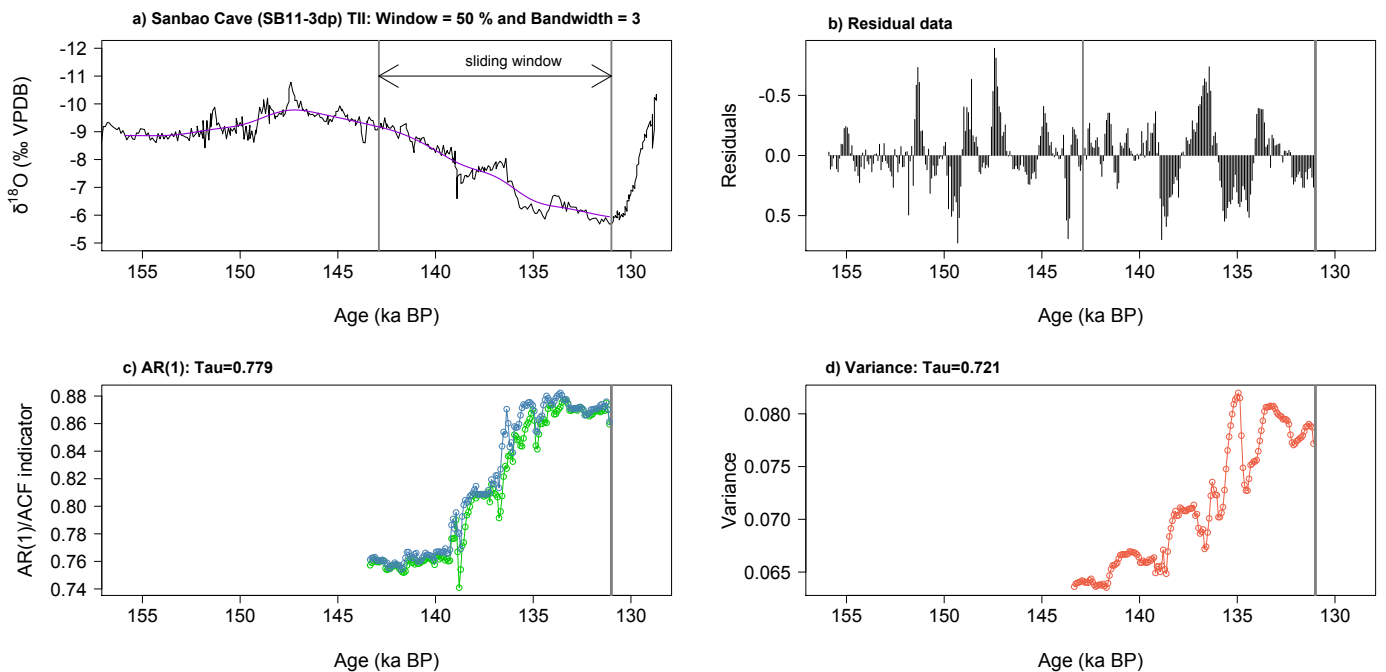

Figure 6. Tipping Point analysis on data from Sanbao Cave (Speleothem SB11; $31^{\circ} 40^{\prime} \mathrm{N}, 110^{\circ} 26^{\prime} \mathrm{E}$ ). (a) Data were smoothed over an appropriate bandwidth (purple line) to produce data residuals (b) and analysed over a sliding window (of size between the two grey vertical lines). The grey vertical line at $131 \mathrm{ka}$ BP indicates the tipping point and the point up to which the data are analysed. (c) AR(1) values and associated Kendall tau value, and (d) displays the variance and associated Kendall tau value.
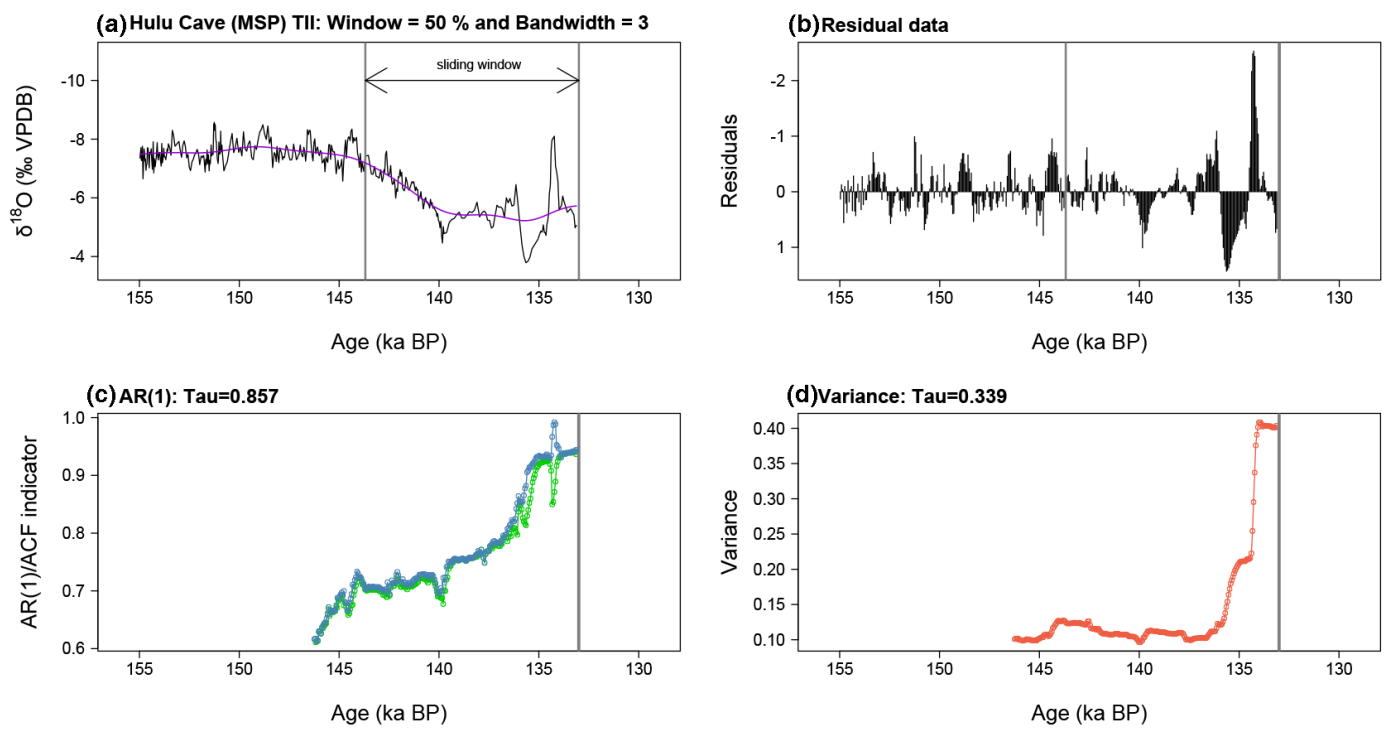

Figure 7. Tipping Point analysis on data from Hulu Cave (Speleothem MSP; $32^{\circ} 30^{\prime} \mathrm{N}, 119^{\circ} 10^{\prime} \mathrm{E}$ ); (a) Data were smoothed over an appropriate bandwidth (purple line) to produce data residuals (b), and analysed over a sliding window (of size between the two grey vertical lines). The grey vertical line at $131 \mathrm{ka} \mathrm{BP}$ indicates the tipping point, and the point up to which the data are analysed. (c) Autocorrelation values and associated Kendall tau value, and (d) the variance and associated Kendall tau value.

Furthermore, a sensitivity analysis was performed (results shown for data preceding the monsoon termination in both speleothem SB11 and MSP, Fig. 8) to ensure that the results are robust over a range of parameters by running repeats of the analysis with a range of smoothing bandwidths used to detrend the original data $(5-15 \%$ of the time series length) and sliding window sizes in which indicators are estimated (25-75\% of the time series length). The colour contours show how the Kendall tau values change when using different parameter choices; for the autocorrelation at Sanbao Cave the Kendall tau values are over 0.8 for the vast majority of smoothing bandwidth and sliding window sizes (Fig. 8a), indicating a robust analysis.

\subsection{Potential model simulations}

To help interpret these results we applied our potential model. In the model we find transitions occur under direct 

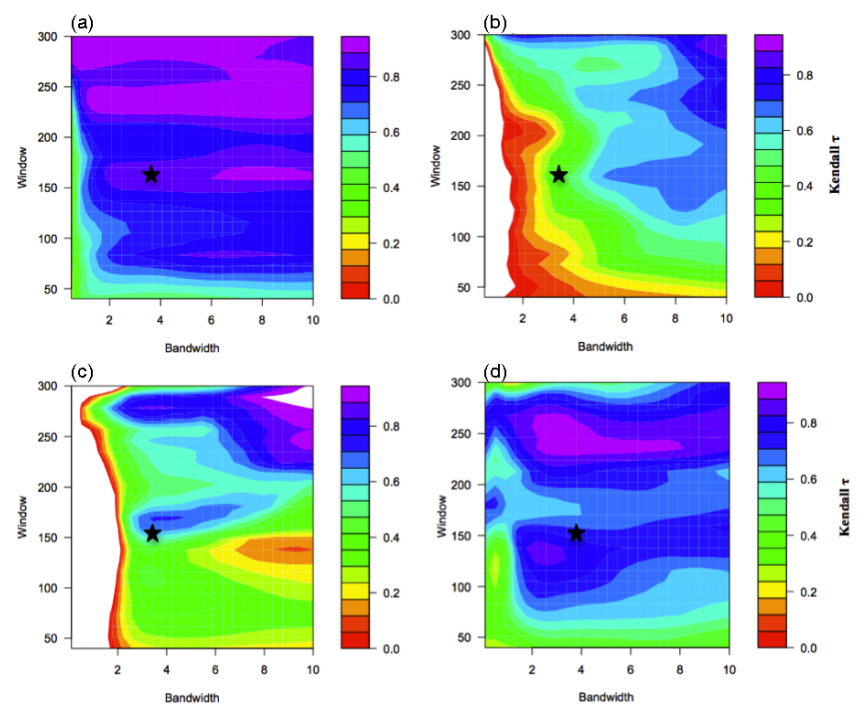

Figure 8. Contour plots showing a range of window and bandwidth sizes for the analysis; (a) Sanbao SB11 autocorrelation, (b) Sanbao SB11 variance, (c) Hulu MSP autocorrelation, (d) Hulu MSP variance. Black stars indicate the parameters used for the analysis in Figs. 6 and 7.

solar insolation forcing when reaching the end of the stable branches, explaining the high degree of synchronicity between the transitions and solar forcing. The 100 realizations produced from our potential model, all initialized at the first data point, appear broadly to follow the path of June insolation at $30^{\circ} \mathrm{N}$ with a small phase lag (Fig. 9). The model simulations also follow the speleothem palaeodata for all but the monsoon transition at $129 \mathrm{ka}$ BP near Termination II, where the model simulations show no extended lag with respect to the insolation. Again it has to be kept in mind that the potential model as a skeleton model can only be expected to qualitatively reproduce the main features of the data. Actually observing the speleothem record as a realization of the model will always be highly unlikely with any model as simple as the present one.

No consistent early warning signals were found in the initial 100 model simulations during the period 224-128 kyr. In order to detect critical slowing down on the approach to a bifurcation, the data must capture the gradual flattening of the potential well. We suggest that early warning signals were not detected due to a relatively fast rate of forcing compared to the sampling of the system; this comparatively poor sampling prevents the gradual flattening of the potential well from being recorded in the data; a feature common to many palaeoclimate data sets. Figure 10 illustrates the different flattening of the potential well over a transition cycle during the glacial period and over the transition cycle at the termination. There is more visible flattening in the potential at the termination, as seen in panel c, which is thought to be due to the reduced amplitude of the solar forcing at the termination.

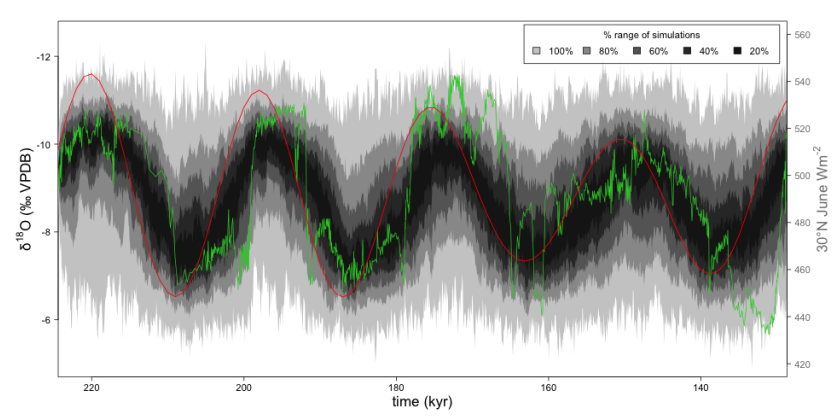

Figure 9. Probability range of 100 model simulations, with the June $30^{\circ} \mathrm{N}$ NHSI (in red), and the palaeodata from SB11 (in green).

The distinction between these two transition cycles helps to explain why early warning signals in the form of increasing autocorrelation and variance are found immediately preceding the termination, but not for the other monsoon transitions.

To test the effect on the early warning signals of the sampling resolution of the model, we compared a range of different sampling time steps in the model (see Sect. 2.4) measuring the Kendall tau values of autocorrelation and variance over each realization of the model (one realization displayed in Fig. 11), which demonstrates the effects of increasing the sampling time step in the model. We found that whereas an increasing sampling time step produces a steady decrease in the Kendall tau values for autocorrelation (Fig. 11b), Kendall tau values remain fairly constant for variance (Fig. 11c), suggesting that the latter is not affected by time step changes. This supports the contention by Dakos et al. (2012b) that "high resolution sampling has no effect on the estimate of variance". In addition, we manipulated the noise level and found that decreasing the noise level by a factor of 2 was necessary to identify consistent early warning signals. This is illustrated in Fig. 11a, where the grey line represents the noise level as determined by the model, which does not follow a step transition, and cannot be adequately detrended by the equation derived from the model. However, once the noise level is sufficiently reduced, early warning signals (displayed here as high Kendall tau values for autocorrelation and variance) can be detected.

\section{Discussion}

It is important to note here that although the detection of early warning signals in time series data has been widely used for the detection of bifurcations in a range of systems (Dakos et al., 2008), there are instances when critical slowing down cannot be detected and/or recorded prior to a bifurcation. First is the assumption that the abrupt monsoon shifts are characterized by a bifurcation, rather than noise-induced tipping or stochastic resonance. The bifurcation hypothesis is supported by previous studies (Zickfeld et al., 2005; Levermann et al., 2009; Schewe et al., 2012) as well as our poten- 


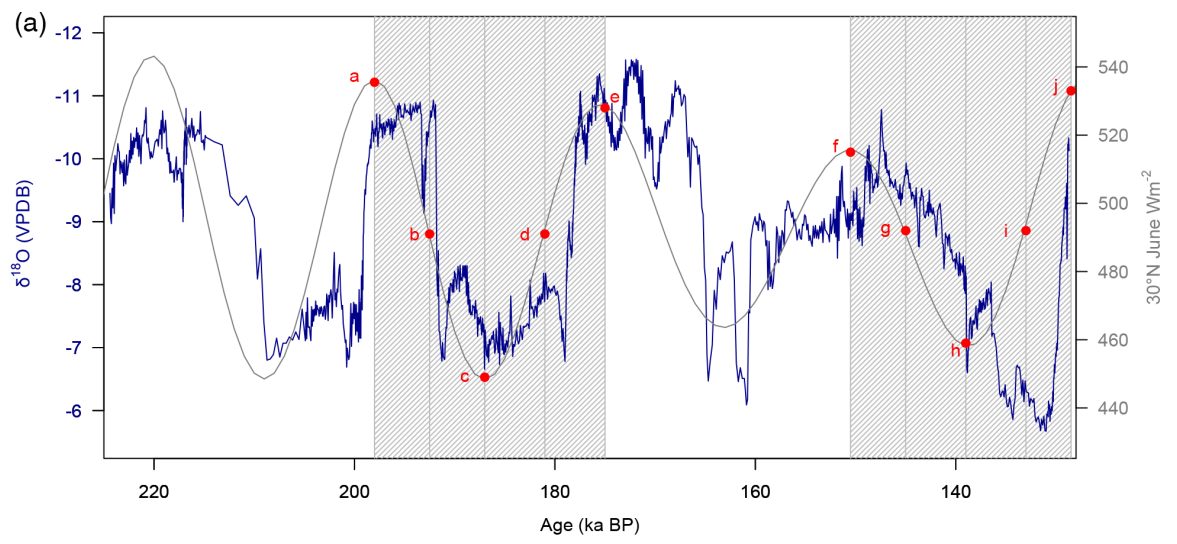

(b)

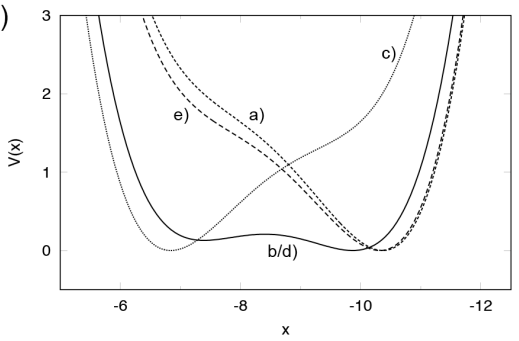

(c)

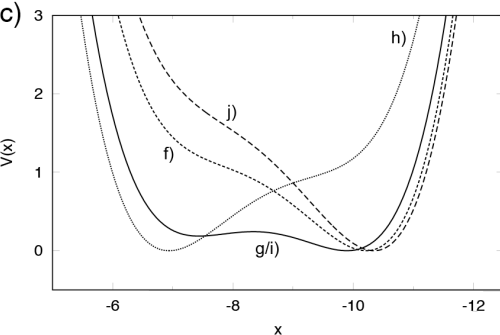

b)

…-..- a) $198 \mathrm{ka}$

— b/d) $192.5 / 181 \mathrm{ka}$

c) $187 \mathrm{ka}$

e) $175 \mathrm{ka}$

c)

f) $150.5 \mathrm{ka}$

g/i) $145 / 133 \mathrm{ka}$

h) $139 \mathrm{ka}$

j) $128.5 \mathrm{ka}$

Figure 10. Potential analysis from the Sanbao $\delta^{18} \mathrm{O}$ data showing the changing shape of the potential over (b) a transition cycle during the glacial period (198-175 kyr); and (c) the transition cycle at the termination (150-128.5 kyr). The potential is shown for stages of the transition over high, medium, and low insolation values, as depicted in panel (a).

tial model, which selects a bifurcation as the most likely scenario (whilst considering noise-induced tipping and stochastic resonance). In a noise-induced tipping or stochastic resonance scenario, no early warning signals would be expected since there would be no gradual change in the stability of the system (Lenton, 2011). Even within the bifurcation scenario, it is possible that early warning signals may not be detected due to external dynamics of the system, such as a high level of stochastic noise, or when there is an insufficient sampling resolution. The results illustrated in Fig. 11 confirm that early warning signals may not be detected for bifurcations if the rate of forcing is too fast compared to the sampling rate, such that the flattening of the potential is poorly recorded in time series; Fig. 11b clearly illustrates the detrimental effect of a lower resolution on Kendall tau values, particularly for autocorrelation. "Missed alarms" may therefore be common in palaeodata where there is an insufficient sampling resolution to detect the flattening of the potential; a high sampling resolution is thus recommended to help avoid this issue. There is more flattening visible in the potential for the monsoon transition at $129 \mathrm{ka}$ BP (Termination II), which is due to the reduced amplitude of the orbital forcing at the termination, but it is unclear whether this is sufficient to explain the early warning signal detected in the palaeodata. We suggest that additional forcing mechanisms may be driving the termination e.g. (Caley et al., 2011) which cannot be captured by the potential model (as evidenced by the trajectory of the data falling outside the probability range of the potential model; Fig. 9).

One possible reason for the detection of a critical slowing down immediately prior to the termination (129 ka BP) is a change in the background state of the climate system. Termination II is preceded by a Weak Monsoon Interval (WMI) in the EASM at 135.5-129 kyr (Cheng et al., 2009), characterized by the presence of a longer lag between the change in insolation and the monsoon transition. The WMI is thought to be linked to migrations in the Inter-tropical Convergence Zone (ITCZ; Yancheva et al., 2007). Changes in the latitudinal temperature gradient (Rind, 1998) or planetary wave patterns (Wunsch, 2006) driven by continental ice volume (Cheng et al., 2009) and/or sea ice extent (Broccoli et al., 2006) have been suggested to play a role in causing this shift in the ITCZ. For instance, the cold anomaly associated with Heinrich event 11 (at $135 \mathrm{ka} \mathrm{BP}$ ) has been invoked as a possible cause of the WMI, cooling the North Atlantic and shifting the Polar Front and Siberian High southwards, forcing an equatorward migration of westerly airflow across Asia (Broecker et al., 1985; Cheng et al., 2009; Cai et al., 2015). Such a scenario would have maintained a low thermal gradient between the land and sea, causing the Weak Monsoon Interval and potentially suppressing a simple insolation response. The implication is that during the earlier monsoon transitions in Stage 6, continental ice volume and/or sea-ice 


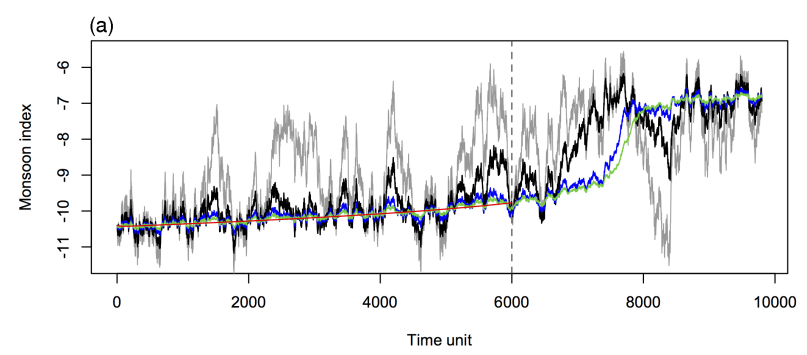

(b) Autocorrelation Kendall tau

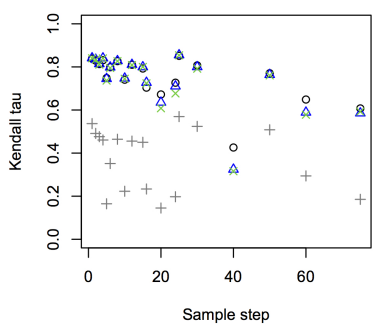

(c) Variance Kendall tau

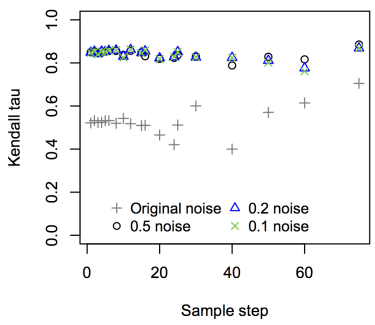

Figure 11. (a) Example of single realization of the approach to a bifurcation from our potential model, which has been generated using four different noise levels (original noise $=$ grey, 0.5 noise $=$ black, 0.2 noise $=$ blue, 0.1 noise $=$ green). Tipping point analysis was applied on each realization, where the red line depicts the detrending line and the grey dashed vertical line is the cut-off point where data are analysed up to; distribution of Kendall tau values for (b) autocorrelation and (c) variance over increasing sample step and differing noise levels.

extent was less extensive than during the WMI, allowing the solar insolation response to dominate.

\section{Conclusions}

We analysed two speleothem $\delta^{18} \mathrm{O}$ records from China over the penultimate glacial cycle as proxies for the past strength of the EASM to test whether we could detect early warning signals of the transitions between the strong and weak regimes. After determining that the data were bimodal, we derived a non-stationary potential model directly from this data featuring a fold bifurcation structure. We found evidence of critical slowing down before the abrupt monsoon shift at Termination II (129 ka BP) in the speleothem $\delta^{18} \mathrm{O}$ data. However, we did not find consistent early warning signals of a bifurcation for the abrupt monsoon shifts in the period between 224-150 kyr, which we term "missed alarms". Exploration of sampling resolution from our model suggests that the absence of robust critical slowing down signals in the palaeodata is due to a combination of rapid forcing and the insufficient sampling resolution, preventing the detection of the steady flattening of the potential that occurs before a bifurcation. We also find that there is a noise threshold at which early warning signals can no longer be detected. We suggest that the early warning signal detected at Termination
II in the palaeodata is likely due to the longer lag during the Weak Monsoon Interval, linked to cooling in the North Atlantic. This allows a steadier flattening of the potential associated with the stability of the EASM and thus enables the detection of critical slowing down. Our results have important implications for identifying early warning signals in other natural archives, including the importance of sampling resolution and the background state of the climate system (full glacial vs. termination). In addition, it is advantageous to use archives which record multiple transitions, rather than a single shift, such as the speleothem records reported here; the detection of an early warning signal during one transition compared to previous events in the same record provides an insight into changing and/or additional forcing mechanisms.

Acknowledgements. We thank Sue Rouillard from the drawing office in the Geography department at the University of Exeter for drawing Fig. 2. T. M. Lenton's contribution was supported by a Royal Society Wolfson Research Merit Award.

The data used in this paper are available from the $\mathrm{Na}$ tional Climate Data Centre, NOAA (SB11: http://hurricane. ncdc.noaa.gov/pls/paleox/f?p=519:1:::::P1_STUDY_ID:8641 and Hulu: http://hurricane.ncdc.noaa.gov/pls/paleox/f?p=519:1:::::P1_ STUDY_ID:5426)

The authors declare no competing financial interests.

Edited by: M. Claussen

\section{References}

An, Z.: The history and variability of the East Asian paleomonsoon climate, Quaternary Sci. Rev., 19, 171-187, 2000.

An, Z., Guoxiong, W., Jianping, L., Youbin, S., Yimin, L., Weijian, Z., Yanjun, C., Anmin, D., Li, L., Jiangyu, M., Hai, C., Zhengguo, S., Liangcheng, T., Hong, Y., Hong, A., Hong, C., and Juan, F.: Global Monsoon Dynamics and Climate Change, Annu. Rev. Earth Planet. Sci., 43, 1-49, 2015.

Ashwin, P., Wieczorek, S., Vitolo, R., and Cox, P.: Tipping points in open systems: bifurcation, noise-induced and rate-dependent examples in the climate system, Philos. T. R. Soc. A, 370, 11661184, 2012.

Baker, A. J., Sodemann, H., Baldini, J. U. L., Breitenbach, S. F. M., Johnson, K. R., Hunen, J. Van, Pingzhong, Z., van Hunen, J., and Pingzhong, Z.: Seasonality of westerly moisture transport in the East Asian Summer Monsoon and its implications for interpreting precipitation $\delta 18 \mathrm{O}$, J. Geophys. Res.-Atmos., 120, 5850-5862, doi:10.1002/2014JD022919, 2015.

Berger, A. and Loutre, M. F.: Insolation values for the climate of the last 10 million years, Quaternary Sci. Rev., 10, 297-317, 1991.

Boulton, C. A. and Lenton, T. M.: Slowing down of North Pacific climate variability and its implications for abrupt ecosystem change, P. Natl. Acad. Sci. USA, 112, 11496-11501, doi:10.1073/pnas.1501781112, 2015.

Broccoli, A. J., Dahl, K. A., and Stouffer, R. J. Response of the ITCZ to Northern Hemisphere cooling, Geophys. Res. Lett., 33, $1-4,2006$. 
Broecker, W. S., Peteet, D. M., and Rind, D.: Does the oceanatmosphere system have more than one stable mode of operation?, Nature, 315, 21-26, 1985.

Cai, Y., Fung, I. Y., Edwards, R. L., An, Z., Cheng, H., Lee, J.-E., Tan, L., Shen, C.-C., Wang, X., Day, J. A., Zhou, W., Kelly, M. J., and Chiang, J. C. H.: Variability of stalagmite-inferred Indian monsoon precipitation over the past 252000 y, P. Natl. Acad. Sci. USA, 112, 2954-2959, 2015.

Caley, T., Malaizé, B., Revel, M., Ducassou, E., Wainer, K., Ibrahim, M., Shoeaib, D., Migeon, S., and Marieu, V.: Orbital timing of the Indian, East Asian and African boreal monsoons and the concept of a "global monsoon", Quaternary Sci. Rev., 30, 3705-3715, 2011.

Cheng, H., Edwards, R. L., Wang, Y., Kong, X., Ming, Y., Kelly, M. J., Wang, X., Gallup, C. D., and Liu, W.: A penultimate glacial monsoon record from Hulu Cave and two-phase glacial terminations, Geology, 34, 217-220, doi:10.1130/G22289.1, 2006.

Cheng, H., Edwards, R. L., Broecker, W. S., Denton, G. H., Kong, X., Wang, Y., Zhang, R., and Wang, X.: Ice Age Terminations, Science, 80, 248-252, 2009.

Cheng, H., Sinha, A., Wang, X., Cruz, F. W., and Edwards, R. L.: The Global Paleomonsoon as seen through speleothem records from Asia and the Americas, Clim. Dynam., 39, 1045-1062, 2012.

Dakos, V., Scheffer, M., van Nes, E. H., Brovkin, V., Petoukhov, V., and Held, H.: Slowing down as an early warning signal for abrupt climate change, P. Natl. Acad. Sci. USA, 105, 14308-12, 2008.

Dakos, V., Carpenter, S. R., Brock, W. A., Ellison, A. M., Guttal, V., Ives, A. R., Kéfi, S., Livina, V., Seekell, D. A., van Nes, E. H., and Scheffer, M.: Methods for detecting early warnings of critical transitions in time series illustrated using simulated ecological data, PLoS One, 7, e41010, doi: 10.1371/journal.pone.0041010, 2012a.

Dakos, V., van Nes, E. H., D'Odorico, P., and Scheffer, M.: Robustness of variance and autocorrelation as indicators of critical slowing down, Ecology, 93, 264-271, 2012b.

Dakos, V., Carpenter, S. R., van Nes, E. H., and Scheffer, M.: Resilience indicators: prospects and limitations for early warnings of regime shifts, Philos. T. R. Soc. B, 370, 20130263, doi:10.1098/rstb.2013.0263, 2014.

Dansgaard, W.: Stable isotopes in precipitation, Tellus, 4, 436-468, 1964.

Ditlevsen, P. D. and Johnsen, S. J.: Tipping points: Early warning and wishful thinking, Geophys. Res. Lett., 37, L19703, doi:10.1029/2010GL044486, 2010.

Donges, J. F., Donner, R. V., Marwan, N., Breitenbach, S. F. M., Rehfeld, K., and Kurths, J.: Non-linear regime shifts in Holocene Asian monsoon variability: potential impacts on cultural change and migratory patterns, Clim. Past, 11, 709-741, doi:10.5194/cp11-709-2015, 2015.

Duan, F., Liu, D., Cheng, H., Wang, X., Wang, Y., Kong, X., and Chen, S.: A high-resolution monsoon record of millennial-scale oscillations during Late MIS 3 from Wulu Cave, south-west China, J. Quaternary Sci., 29, 83-90, 2014.

Hartigan, J. A. and Hartigan, P. M.: The Dip Test of Unimodality, Ann. Stat., 13, 70-84, 1985.
Held, H. and Kleinen, T.: Detection of climate system bifurcations by degenerate fingerprinting, Geophys. Res. Lett., 31, L23207, doi:10.1029/2004GL020972, 2004.

Ives, A.: Measuring Resilience in Stochastic Systems, Ecol. Monogr., 65, 217-233, 1995.

Kendall, M. G.: Rank correlation methods, Oxford, Griffen, 1948.

Kleinen, T., Held, H., and Petschel-Held, G.: The potential role of spectral properties in detecting thresholds in the Earth system: application to the thermohaline circulation, Ocean Dynam., 53, 53-63, 2003.

Kutzbach, J. E.: Monsoon climate of the early Holocene: climate experiment with the Earth's orbital parameters for 9000 years ago, Science, 80, 59-61, 1981.

Kwasniok, F.: Predicting critical transitions in dynamical systems from time series using nonstationary probability density modeling, Phys. Rev. E, 88, 052917, 2013.

Kwasniok, F.: Forecasting critical transitions using data-driven nonstationary dynamical modeling, Phys. Rev. E, accepted, 2015.

Kwasniok, F. and Lohmann, G.: Deriving dynamical models from paleoclimatic records: Application to glacial millennial-scale climate variability, Phys. Rev. E, 80, 066104, 2009.

Lee, J.-E. and Swann, A. L.: Evaluation of the "amount effect" at speleothem sites in the Asian monsoon region, IOP Conf. Ser. Earth Environ. Sci., 9, 12023 pp., 2010.

Lenton, T. M.: Early warning of climate tipping points, Nature Climate Change, 1, 201-209, 2011.

Lenton, T. M., Livina, V. N., Dakos, V., van Nes, E. H., and Scheffer, M: Early warning of climate tipping points from critical slowing down: comparing methods to improve robustness, Philos. T. R. Soc. A, 370, 1185-1204, 2012.

Levermann, A., Schewe, J., Petoukhov, V., and Held, H.: Basic mechanism for abrupt monsoon transitions, P. Natl. Acad. Sci. USA, 106, 20572-20577, 2009.

Li, T.-Y., Shen, C.-C., Huang, L.-J., Jiang, X.-Y., Yang, X.-L., Mii, H.-S., Lee, S.-Y., and Lo, L.: Stalagmite-inferred variability of the Asian summer monsoon during the penultimate glacialinterglacial period, Clim. Past, 10, 1211-1219, doi:10.5194/cp10-1211-2014, 2014.

Lisiecki, L. E. and Raymo, M. E.: A Pliocene-Pleistocene stack of 57 globally distributed benthic $\delta^{18} \mathrm{O}$ records, Paleoceanography, 20, 1-17, 2005.

Liu, X., Liu, Z., Kutzbach, J. E., Clemens, S. C., and Prell, W. L.: Hemispheric Insolation Forcing of the Indian Ocean and Asian Monsoon: Local versus Remote Impacts, Am. Meteorol. Soc., 19, 6195-6208, 2006.

Liu, Z., Wen, X., Brady, E. C., Otto-Bliesner, B., Yu, G., Lu, H., Cheng, H., Wang, Y., Zheng, W., Ding, Y., Edwards, R. L., Cheng, J., Liu, W., and Yang, H.: Chinese cave records and the East Asia Summer Monsoon, Quaternary Sci. Rev., 83, 115-128, 2014.

Maher, B. A.: Holocene variability of the East Asian summer monsoon from Chinese cave records: a re-assessment, The Holocene, 18, 861-866, 2008.

Pausata, F. S. R., Battisti, D. S., Nisancioglu, K. H., and Bitz, C. M.: Chinese stalagmite $\delta^{18} \mathrm{O}$ controlled by changes in the Indian monsoon during a simulated Heinrich event, Nat. Geosci., 4, 474-480, 2011

Rind, D.: Latitudinal temperature gradients and climate change, J. Geophys. Res., 103, 5943-5971, 1998. 
Rodionov, S. N.: A sequential algorithm for testing climate regime shifts, Geophys. Res. Lett., 31, L09204, doi:10.1029/2004GL019448, 2004.

Ruddiman, W. F.: What is the timing of orbital-scale monsoon changes?, Quaternary Sci. Rev., 25, 657-658, 2006.

Scheffer, M.: Foreseeing tipping points, Nature, 467, 6-7, 2010.

Schewe, J., Levermann, A., and Cheng, H.: A critical humidity threshold for monsoon transitions, Clim. Past, 8, 535-544, doi:10.5194/cp-8-535-2012, 2012.

Sun, Y., Kutzbach, J., An, Z., Clemens, S., Liu, Z., Liu, W., Liu, X., Shi, Z., Zheng, W., Liang, L., Yan, Y., and Li, Y.: Astronomical and glacial forcing of East Asian summer monsoon variability, Quaternary Sci. Rev., 115, 132-142, 2015.

Thompson, J. and Sieber, J.: Predicting climate tipping as a noisy bifurcation: a review, Int. J. Bifurc. Chaos, 21, 399-423, 2011.

Wang, H. and Chen, H.: Climate control for southeastern China moisture and precipitation: Indian or East Asian monsoon?, J. Geophys. Res. Atmos., 117, D12109, doi:10.1029/2012JD017734, 2012.

Wang, Y., Cheng, H., Edwards, R. L., Kong, X., Shao, X., Chen, S., Wu, J., Jiang, X., Wang, X., and An, Z.: Millennial- and orbitalscale changes in the East Asian monsoon over the past 224000 years, Nature, 451, 18-21, 2008.
Wang, P.: Global monsoon in a geological perspective, Chinese Sci. Bull., 54, 1113-1136, doi:10.1007/s11434-009-0169-4, 2009.

Wang, Y. J., Cheng, H., Edwards, R. L., An, Z. S., Wu, J. Y., Shen, C. C., and Dorale, J. A: A high-resolution absolute-dated late Pleistocene Monsoon record from Hulu Cave, China, Science, 80, 2345-2348, 2001.

Wu, G., Liu, Y., He, B., Bao, Q., Duan, A., and Jin, F.-F.: Thermal controls on the Asian summer monsoon, Sci. Rep., 2, 1-7, 2012.

Wunsch, C.: Abrupt climate change: An alternative view, Quaternary Res., 65, 191-203, 2006.

Yancheva, G., Nowaczyk, N. R., Mingram, J., Dulski, P., Schettler, G., Negendank, J. F. W., Liu, J., Sigman, D. M., Peterson, L. C., and Haug, G. H.: Influence of the intertropical convergence zone on the East Asian monsoon, Nature, 445, 74-77, 2007.

Yuan, D., Cheng, H., Edwards, R. L., Dykoski, C. A., Kelly, M. J., Zhang, M., Qing, J., Lin, Y., Wang, Y., Wu, J., Dorale, J. A., An, Z., and Cai, Y.: Timing, duration, and transitions of the last interglacial Asian monsoon, Science, 80, 575-578, 2004.

Zickfeld, K., Knopf, B., Petoukhov, V., and Schellnhuber, H. J.: Is the Indian summer monsoon stable against global change?, Geophys. Res. Lett., 32, L15707, doi:10.1029/2005GL022771, 2005. 